\title{
机械驱动下 $\mathrm{Fe}-\mathrm{Sn}$ 及 $\mathrm{Cu}-\mathrm{Sn}$ 纳米 高温相的形成
}

\author{
杨元政 ${ }^{(1)}$ 马学鸣 ${ }^{(3)}$ 董远达 ${ }^{(3)}$ 庄育智 ${ }^{(8)}$ \\ (1) 华南理工大学机械工程二系,广州 510641; (2) 中国科学院金属研究所, 沈阳 110015; (3) 上海工业大学 \\ 材料科学与工程系, 上海 200072)
}

\section{关链词机械合金化、纳米合金、过饱和固溶体}

通常的块体材料由具有长程序的晶态组元或短程序的非晶态组元构成, 因而其体内存在 的界面是很少的. 纳米材料因其组元颗粒细小, 处于界面上的原子既没有长程序也没有短 程序, 从而界面成为其基本组元, 不容忽略. 由此引起了纳米材料许多不同于通常块体材料 的独特性能 ${ }^{[1,2]}$. 然而纳米材料的制备一般局限于惰性气体冷凝沉积技术 ${ }^{[3]}$ 和液态化学方法 ${ }^{[4]}$. 近来, 机械合金化法也成功地制备了多种金属基纳米新型材料 ${ }^{[5-\eta}$. 从而使其成为制备纳米材 料的一种有效的固态方法. 本文报道用机械合金化法制备 $\mathrm{Fe} S \mathrm{Sn}$ 和 $\mathrm{Cu}-\mathrm{Sn}$ 高温纳米合金.

所用 $\mathrm{Fe}$ 粉、 $\mathrm{Cu}$ 粉和 $\mathrm{Sn}$ 粉的平均粒径为 $75 \mu \mathrm{m}$, 纯度大于 $99 \%$. 样品按所需原子比混 合. 球与粉的重量比约为 10:1. X 射线衍射实验在 Philips PW1700 X 射线衍射仪上进行, 电 压为 $40 \mathrm{kV}$, 电流为 $40 \mathrm{~mA}$, 采用 $\mathrm{Cuk} \alpha$ 辐射 $(\lambda=1.54 \dot{\mathrm{A}})$. 粒径测量采用步进扫描, 步长为每 $4 \mathrm{~s} 0.06^{\circ}$. 热分析在 Perkin-Elmer DSC-2C 上进行.

图 1 是 $\mathrm{Cu}-\mathrm{Sn}$ 样品在球磨 $100 \mathrm{~h}$ 之后的 $\mathrm{X}$ 射线衍射谱. 样品 $\mathrm{Cu}_{90} \mathrm{Sn}_{10}$ 的衍射谱中没有 $\mathrm{Sn}$ 的衍射峰, 仅存在宽化了的 $\mathrm{Cu}$ 衍射峰. 用 $\mathrm{X}$ 衍射线形分析测得的粒径大小为 $23 \mathrm{~nm}$, 可见经 球磨后样品中颗粒已经达到纳米尺寸. 在纳米尺度下, 原子的扩散变得相当容易, 同时界面 上的各个原子的化学势将发生变化, 为不同原子的相互扩散、固溶和键合提供了可能 ${ }^{[8}$. 经物 相分析 $\mathrm{Cu}_{50} \mathrm{Sn}_{50}$ 和 $\mathrm{Cu}_{70} \mathrm{Sn}_{30}$ 样品的衍射谱中 $\mathrm{Cu}$ 和 $\mathrm{Sn}$ 的衍射峰都 已消失, $\mathrm{Cu}_{70} \mathrm{Sn}_{30}$ 样品中出现 的主要是 $\mathrm{Cu}-\mathrm{Sn}$ 的 $\varepsilon$ 相; 而 $\mathrm{Cu}_{50} \mathrm{Sn}_{50}$ 样品中出现的主要则是 $\mathrm{Cu}-\mathrm{Sn}$ 的 $\eta$ 相. 可见, 机械合金化 在室温下不仅能扩展 Cusn 的固溶范围, 而且能得到该体系的高温合金, 即 $\mathrm{Cu}-\mathrm{Sn}$ 的 $\eta$ 相和 $\varepsilon$ 相. 图 2 显示了样品在升温速率为 $40 \mathrm{~K} / \mathrm{min}$ 下的 DSC (Differential scanning calorimetry) 曲 线. 在升温过程中, $\mathrm{Cu}_{90} \mathrm{Sn}_{10}$ 样品先是两个宽大放热峰, 然后是吸热峰. 第一个放热峰对应固 溶体内的应力松驰, 第二个放热峰则对应于部分溶质原子析出和晶粒长大的过程. 而宽化吸 热峰则对应于混合物中 $\mathrm{Sn}$ 的熔融峰 ${ }^{[9]}$. 样品 $\mathrm{Cu}_{50} \mathrm{Sn}_{50}$ 和 $\mathrm{Cu}_{70} \mathrm{Sn}_{30}$ 的 DSC 曲线形状的解释也是 类似的, 即两个放热峰是对应于应力释放和高温相的晶粒长大. 在样品 $\mathrm{Cu}_{50} \mathrm{Sn}_{50}$ 中, 吸热峰位 置在 $740 \mathrm{~K}$ 左右则对应于 $\eta$ 相的相变, 而在样品 $\mathrm{Cu}_{70} \mathrm{Sn}_{30}$ 中, 在 DSC 测量的温度范围内最大吸 热峰位置尚未出现, 由相图可知 $\varepsilon$ 相变位置在 $950 \mathrm{~K}$ 左右 ${ }^{[9]}$.

1993-12-15 收稿, 1994-04-09 收修改稿. 


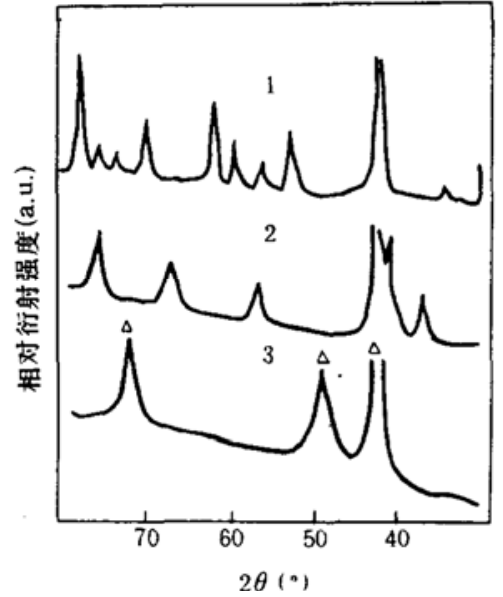

图 1 球磨 $100 \mathrm{~h}$ 后 $\mathrm{Cu}-\mathrm{Sn}$. 样品的 $\mathrm{X}$ 射线衍射谱 1 为 $\mathrm{Cu}_{50} \mathrm{Sn}_{90}, 2$ 为 $\mathrm{Cu}_{70} \mathrm{Sn}_{30}, 3$ 为 $\mathrm{Cu}_{90} \mathrm{Sn}_{10}$

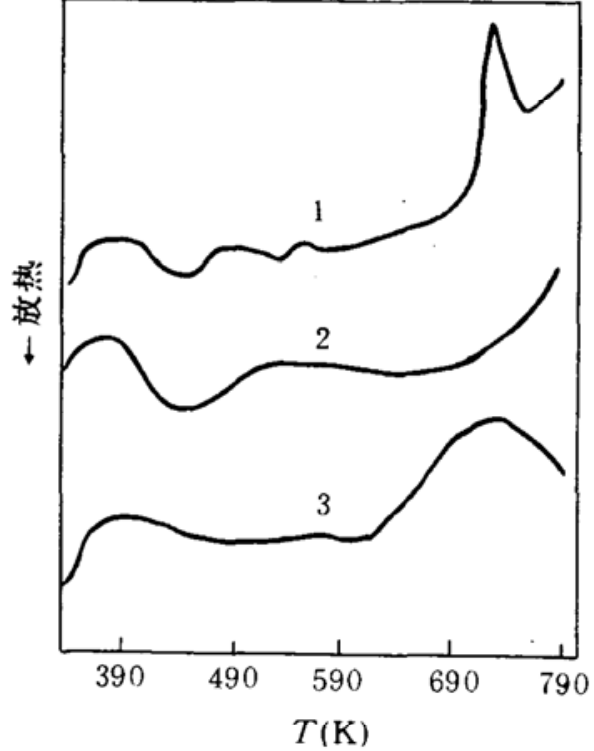

图 2 球磨后 $\mathrm{Cu}-\mathrm{Sn}$ 样品在升温速率为 $40 \mathrm{~K} / \mathrm{min}$ 下的 DSC 曲线

1 为 $\mathrm{Cu}_{50} \mathrm{Sn}_{50}, 2$ 为 $\mathrm{Cu}_{30} \mathrm{Sn}_{30}, 3$ 为 $\mathrm{Cu}_{90} \mathrm{Sn}_{10}$

图 3 是 $\mathrm{Fe}-\mathrm{Sn}$ 样品球磨 $120 \mathrm{~h}$ 后的 $\mathrm{X}$ 射线衍射谱. 在含 $\mathrm{Sn}$ 量不大于 $40 \mathrm{at} \%$ 的情况下, 不存 在 $\mathrm{Sn}$ 的衍射峰, 仅有宽化了的 $\mathrm{Fe}$ 衍射峰, 即样品形成具有 $\mathrm{Fe}$ 结构的过饱和固溶体. 其中对 $\mathrm{Fe}_{20} \mathrm{Sn}_{30}$ 样品进行 $\mathrm{X}$ 射线衍射线形分析, 磨后的样品中平均颗粒大小为 $16 \mathrm{~nm}$. 即在纳米尺度 下 $\mathrm{Fe}-\mathrm{Sn}$ 的溶解度得到了极大的扩展. 在样品 $\mathrm{Fe}_{50} \mathrm{Sn}_{50}$ 中, $\mathrm{Fe}$ 与 $\mathrm{Sn}$ 的衍射峰都已消失, 经物 相分析得知, 球磨后的产物为 $\mathrm{Fe}_{1.3} \mathrm{Sn}$ 和 $\mathrm{FeSn}_{2}$, 即形成了金属间化合物. 可见用机械合金化的 方法能方便地制备 $\mathrm{Fe}-\mathrm{Sn}$ 的高温相 $\mathrm{Fe}_{1.3} \mathrm{Sn}$ 和 $\mathrm{FeSn}_{2}$, 而不是 $\mathrm{Fe}_{50} \mathrm{Sn}_{50}$ 相. 图 4 给出的是 $\mathrm{Fe}-\mathrm{Sn}$ 部分样品在升温速率为 $40 \mathrm{~K} / \mathrm{min}$ 下的 DSC 曲线. 可见, 在升温过程中, 所有样品先是两个宽 大的放热峰, 然后是吸热峰. 对于两个放热峰的解释与图 2 是完全类似的, 即分别对应于球磨

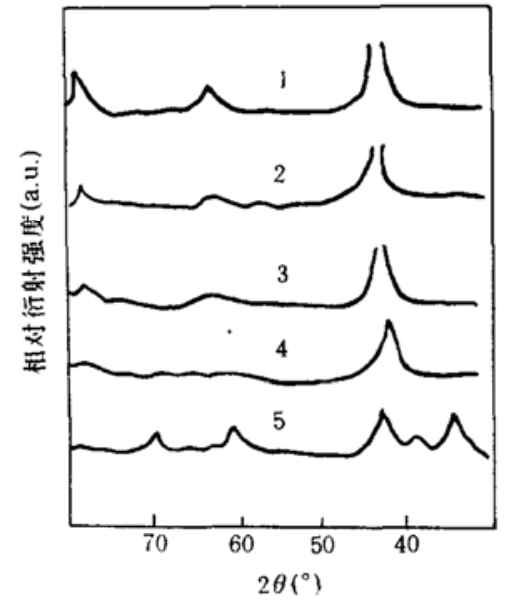

图 3 球磨 $120 \mathrm{~h}$ 后 $\mathrm{FeSn}$ 样品的 X 射线衍射谱 1 为 $\mathrm{Fe}_{90} \mathrm{Sn}_{10}, 2$ 为 $\mathrm{Fe}_{80} \mathrm{Sn}_{20}, 3$ 为 $\mathrm{Fe}_{20} \mathrm{Sn}_{30}, 4$ 为 $\mathrm{Fe}_{60} \mathrm{Sn}_{40}, 5$ 为 $\mathrm{Fe}_{50} \mathrm{Sn}_{50}$

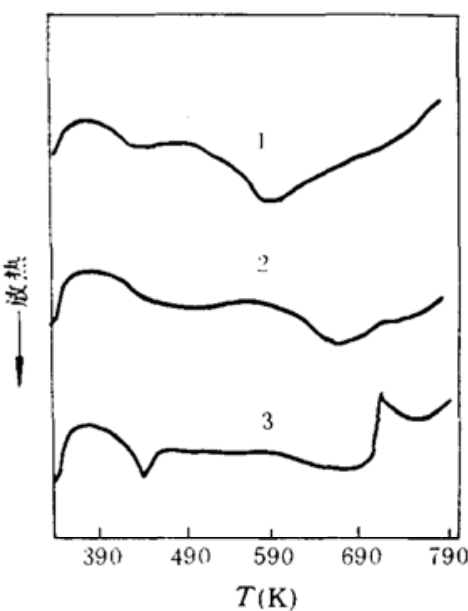

图 $4 \mathrm{Fe}-\mathrm{Sn}$ 部分样品在升温速率为 $40 \mathrm{~K} / \mathrm{min}$ 下 的 DSC 曲线

1 为 $\mathrm{Fe}_{50} \mathrm{Sn}_{90}, 2$ 为 $\mathrm{Fe}_{70} \mathrm{Sn}_{30}, 3$ 为 $\mathrm{Fe}_{90} \mathrm{Sn}_{10}$ 
样品的应力松驰和部分溶质原子析出或晶粒长大的过程. 此外, 样品 $\mathrm{Fe}_{90} \mathrm{Sn}_{10}$ 的 DSC 曲线上 存在一个吸热峰, 它对应于固溶体分解之后混合物中 $\mathrm{Sn}$ 的熔融峰. 由于对等成分 $\mathrm{Fe}_{50} \mathrm{Sn}_{30}$ 为 金属间化合物混合相, 且相变温度都比较高 ${ }^{[10}$, 因此还没有出现明显的吸热峰. 样品 $\mathrm{Fe}_{70} \mathrm{Sn}_{30}$ 的 DSC 曲线变化介于两者之间, 存在一个 Sn 熔融峰的台阶.

实验结果表明: 机械合金化能使体系的颗粒极大地细化, 从而在纳米尺度下扩展 Fe-Sn 和 $\mathrm{Cu}$ Sn 的固溶度, 形成过饱和固溶体; 在某些适当的成分附近形成金属化合物, 即体系的高温 相. 在 Fe-Sn 体系中, $\mathrm{Sn}$ 在 $\mathrm{Fe}$ 中的固溶度可达到含 $\mathrm{Sn}$ 量为 $40 \mathrm{at} \%$, 在对等成分时能形成 $\mathrm{Fe}_{1.3} \mathrm{Sn}$ 和 $\mathrm{FeSn}_{2}$ 金属合金. 而在 $\mathrm{Cu}-\mathrm{Sn}$ 体系中, $\mathrm{Sn}$ 在 $\mathrm{Cu}$ 中的固溶度同样得到扩展, 在成分为 $\mathrm{Cu}_{x 0} \mathrm{Sn}_{30}$ 和 $\mathrm{Cu}_{50} \mathrm{Sn}_{30}$ 时, 则分别能形成 $\varepsilon$ 相和 $\eta$ 相. 所有样品的热分析结果表明: 两个放热峰对 应于球磨样品体内的应力释放, 固溶分解或晶粒长大, 而吸热峰则对应于高温相变或熔融 峰.

\section{参考文、献}

[1] Nieh, T. G., Wadsworth, J., Scri. Metall., 1991, 25(4):955-958.

[2] Rupp, J., Birringer, R., Phys. Rev., 1987, B36(15): 7888-7890.

[3] Granquist, C. G., Buhrman, R. A., J. Appl. Phys., 1976, 47(5):2200-2219.

[4] Searcy, A. W., J. Am. Ceram. Soc., 1983, 70(1): 155- 161.

[5] Shingu, P. H., Huang, B., Nishitani, S. R. a al., Proc. JIMIS-5 Supplement to Trans. JIM, 1988, 29:3-10.

[6] Hellstern, E., Fecht, H. J., Fu, Z. et al., J. Appl. Phys., 1989, 60(1):305-310.

[7] Yang, Y. Z., Ma, X. M., Dong, Y. D. et al., Chinese Phys. Lett., 1992, 9(5):266-268.

[8] Mutschele, T., Kirchheim, R., Scri. Metall., 1987, 21(2): 135-140.

[9] Massalski, T. B., Binary Alloy Phase Diagrams, American Society for Metak, 1987, 965.

[10] Massakki, T. B., Binary Alloy Phase Diagrams, American Society for Metals, 1987, 1110. 